\title{
What's up in pulmonary sleep medicine?
}

\section{Report from the 2012 ATS (American Thoracic Society) annual conference in San Francisco}

\author{
Nikolaus Netzer
}

Received: 23 May 2012 /Revised: 30 May 2012 / Accepted: 7 June 2012 / Published online: 14 July 2012

(C) Springer-Verlag 2012

The recent developments in sleep medicine, which have political implications on reimbursement issues, are discussed more controversially than ever. It was a good idea by the sleep assembly of the American Thoracic Society (ATS) to schedule a full morning session with pros and cons to topics that seem so clear (if you are in the right party) right in the middle of the conference when weekend attendees and midweek attendees cross their path.

Are home PSGs the future of sleep medicine and is the old sleep lab gone? Will the sleep patient of the future be a distant person, who we never will meet face to face again and more an anonymous data file with EEGs and respiratory curves. Sam Kuna (PA, USA) spoke the pro part and listening to his speech was no different in causing cold shivers and goose bumps for some of the experienced sleep physicians than watching the last part of the "Final Destination" series in a dark movie theater. Of course Sam listed all the good reasons like "less expensive", "no extra room and energy cost for the health care provider", "no first night effects", "inclusion of the spouse in the diagnostic process", and "equally reliable to in-lab polysomnography (PSG)". What a relief for us old guys to hear the calming sentences of Allan Pack (PA, USA) that home studies could never fully replace in-lab PSG with a physician on site to see the patient, continuous positive airway pressure (CPAP) titration by a specialist and someone who can interact if problems occur and electrodes fall off. Experience however tells the story that in the end, the insurances will decide which road will be taken.

\footnotetext{
N. Netzer $(\bowtie)$

Hermann Buhl Institute for Hypoxia and Sleep Medicine Research, Research Program of the Paracelsus Medical University, Salzburg, Ghersburgstr. 9,

83043 Bad Aibling, Germany

e-mail: nikinetzer@yahoo.com
}

Is sleep apnea screening necessary in each patient with a risk for cardiovascular diseases? Virend Sommers (Rochester, MN) defended that statement based on the hundreds of publications from the many studies which have linked sleep disordered breathing to high BP, stroke, metabolic syndrome, and others. Causing smiles and a chuckle in the audience, Virend Sommers showed different statements in publications from his opponent in the discussion, John Stradling (Oxford, UK), who first denied until 2002 in several papers that obstructive sleep apnea (OSA) causes any cardiovascular risks, but became a great defender of the cardiovascular risk linked to OSA in papers from 2003 on. John countered that for screening tests in cardiovascular risk patients, we would need a clear cutoff where to start. This cutoff point would not exist. The Epworth Sleepiness Scale (ESS) would be completely useless in this question. Only if the high apnea-hypopnea index (AHI) is already known, it shows that those with high ESS score have also high BP. There would be no evidence that an early-onset treatment of OSA would reduce mortality by cardiovascular diseases. CPAP would actually reduce mortality but not to a higher level than placebo. And finally treating high BP in OSA patients exclusively with a modern medication like Valsartan and skip CPAP would actually be more effective than the CPAP therapy alone. So there would be no reason for screening people with high BP, etc.

Are MADs (oral appliances) equal to CPAP? Peter Cistulli (Sydney, Australia) argued that while CPAP is more effective in reducing the AHI, the higher adherence of patients to their mandibular advancement devices (MADs; 50 vs. $70 \%$ in most subjective responses) makes up for that in overall efficacy. The time use would be longer so the overall avoidance of apneas more frequent. In many parameters other than AHI reduction like QOL, cognitive function, etc., MADs are in many studies and one just finished with 160 patients in Sydney even slightly better than CPAP. David 
Rappaport (NY, USA) argued against that that MADs in the long term do not really show a better adherence than CPAP, after 12 month both methods are equally up in a recent publication. If patients who had both treatments would be asked confidentially what they think would be best for them they would say: CPAP, but they would maybe prefer MADs to better please their spouses. And finally in regard to cost effectiveness he argued that since CPAP prices have come down a lot, CPAP would now be half as expensive as MADs over a 2-year period.

Are usage-adherence tracking devices in CPAPs useful? Patrick Strollo (PA, USA) stated that the longer the CPAP is used, the more effective the treatment is regarding all possible outcome parameters (EDS, QOL, BP, etc.); therefore, it would be necessary to track the usage time. Studies had clearly shown that there is no correlation between subjectively self-reported usage and objectively measured usage. Medicare and other governmental institutions would now for that reason require the use of the tracking devices, and following the recommendations of overall Health Outcomes as imposed in a major paper in the NEJM in 2010 the control of effectiveness of treatment is a major issue for the whole society. Richard Schwab (PA, USA) countered that several studies, which he actually cited from this journal Sleep and Breathing, had shown that the tracking devices do not work accurately, and none of them would have ever been validated vs. in-lab PSG. Especially that hypopneas would be wrongfully calculated, so would be leakage. If the tracking devices would not be reliable, they would not make real sense and decisions to take the device away from the patient would be based on something doubtful.

Besides the fact that this pro and con session was a little bit Pennsylvania loaded, it was really entertaining.

A major topic in this year's ATS meeting was not surprisingly the new hypoglossal nerve stimulators and the multicenter studies done with the new devices. Three different devices and approaches have been presented by a Cleveland/
German collaboration around Kingman Strohl, a Harvardbased group around Atul Malhotra, and a Belgian group around Daniel Rodenstein. The new devices, heavily improved obviously from their predecessors 15 years ago in sensitive stimulation, seem to be equivalently effective in reducing apneas and hypopneas. Their huge advantage now is that they all do not interfere with sleep anymore. In all studies, sleep even improved with increasing REM and NREM 3. One huge difference in the Belgian study (or at least only that group looked at that) was that the targeted hypoglossal nerve stimulation had a training effect on the pharynx muscles and that apneas did not occur for days even after the device was shut off.

However, the question arises if the operation risk, which goes along with surgery on the neck and the needed central anesthesia, and a certain infection risk of around between 1 and $7 \%$ from the operation in the different studies will make this treatment the treatment of choice for the majority of patients in the future.

An official press release by ATS concerned new results from a long-term analysis of data from the Wisconsin cohort study, which showed a higher risk of cancer after several years in those persons who had been identified with OSA in the cohort group. Finally, there is a potentially new dimension to recognition and management of OSA. New results were presented in abstract form on an association of cancer and higher values of AHI $(>30)$. The Wisconsin cohort analysis of long-term (15 years) outcomes examined mortality and reported a higher risk ( $\sim 5$-fold) of cancer with severe OSA in the original cohort group reported in 1993. The association was stronger ( $\sim 7$-fold) when examined against oxygen saturation profiles. This association is plausible based on animal studies showing greater growth of cancer in experimental animals exposed to intermittent hypoxia. The number of affected individuals was rather small ( $\sim 50)$. Whether these results will hold up in other datasets, and whether causality can be determined, appear as major questions for future research. 